\title{
Tympanic membrane grafting, underlay periosteal versus inlay cartilage grafts: a comparative study
}

\begin{abstract}
Various grafting materials and different ways of positioning have been described for tympanic membrane (TM) reconstruction. However, there are few clinical trials comparing cartilage and periosteal grafts.
\end{abstract}

Aims: A comparison between underlay periosteal grafting (UPG) and inlay cartilage grafting (ICG) of TM.

Material and Methods: A retrospective controlled trial. Group A, included 88 patients underwent UPG. Group B, included 61 patients subjected to ICG.

\begin{abstract}
Assessment included: Mean Air Bone Gap (ABG), operative time and graft take. Data was summarized using mean and standard deviation in quantitative data and using frequency (count) and relative frequency (percentage) for categorical data. Comparisons between quantitative variables were done using unpaired t test. For comparing categorical data, Chi square $\left(\chi^{2}\right)$ test was performed.
\end{abstract}

Results: Graft healing was statistically insignificant between both groups, with group A cases showing $93 \%$ complete graft healing and $92 \%$ in group $\mathrm{B}(\mathrm{P}$ value $=0.8)$. However, group A mean difference $A B G$ was statistically significant ( $-11 \mathrm{~dB} \pm 5 \mathrm{SD}$ ) when compared to group $\mathrm{B}(-9 \mathrm{~dB} \pm 7 \mathrm{SD})$ with $\mathrm{P}$ value $=0.01$. Operative time comparison was statistically significant with 33 minutes $\pm 5 \mathrm{SD}$ in group $\mathrm{A}$, while in group $\mathrm{B}$ was 19 minutes $\pm 3 \mathrm{SD}$ $(\mathrm{P}<0.001)$

Conclusion: Underlay periosteal and inlay cartilage grafts show high rates of graft take, with better hearing results using underlay periosteal grafts but shorter operative time with inlay cartilage grafts. Long term follow up is recommended in future studies.

Keywords: cartilage tympanoplasty, periosteal graft tympanoplasty, type I tympanoplasty, tympanoplasty grafts
Volume 10 Issue 5 - 2018

\begin{abstract}
Ahmed Mahmoud ElBatawi,' Mostafa A ElTaher, ${ }^{2}$ Mohamed Abd Elmottaleb Sabaa'

'ENT Department, Cairo University, Egypt

${ }^{2}$ ENT Department, Sohag University, Egypt
\end{abstract}

Correspondence: Ahmed Mahmoud El Batawi, Lecturer of otorhinolaryngology, Cairo University, Egypt, Tel +201119931237, Email batawi@gmaail.com, Ahmed.elbatawi@cu.edu.eg,Ahmed.elbatawi@kasralainy.edu.eg

Received: February 21, 2018| Published: September 25, 2018

\section{Introduction}

Middle ear infection has been a problem facing the humans; it is as ancient as humanity itself. ${ }^{1}$ Tympanoplasty was introduced by Wullstein \& Zollner ${ }^{2,3}$ in 1952 for closure of the perforated tympanic membrane (TM). Various grafting materials and ways of positioning have been described.

Inlay cartilage myringoplasty has gained consensus by many surgeons for the repair of tympanic membrane erforations through a transcanal approach. ${ }^{4}$ It provides several practical advantages, for example, no support through external auditory canal packing or middle ear is required as the graft has good stability. Postoperatively patient comfort is enhanced, and the operation has less expenditure because of contracted operative and recovery time. ${ }^{5}$

Periosteum of the mastoid cortex has been described in the literature as a suitable grafting material for tympanic membrane repair, with several advantages. ${ }^{6}$ However, there are few clinical trials comparing both types of grafts.

\section{Material and methods}

This study was retrospective controlled trial, comparing two different techniques performed by two university hospitals, in the period from 2014 to 2016. One hundred fourty nine patients were involved in this study. Patients were classified into two groups:

\section{Group A}

Included 88 patients underwent underlay periosteal TM grafting performed in a university hospital.

\section{Group B}

Included 61 patients subjected to inlay cartilage tympanoplasty in another university hospital.

All patients had dry central TM perforation for at least 2 months before surgery. Cases with granulations, discharge, myringitis, active infection, otomycosis, mixed hearing loss, previous failed myringoplasty, and cases which required cortical mastoidectomy, middle ear exploration were excluded.

Preoperative history taking and audiological assessment were done by calculating Air Bone Gap (ABG) as the average hearing level at $500,1000,2000$ and $4000 \mathrm{~Hz}$ frequencies. Intraoperatively the time consumed during the operation was recorded in minutes.

Postoperative evaluation was done 6 months post surgery included: graft take and mean $\mathrm{ABG}$.

\section{Statistical methods}

Data were coded and entered using the statistical package Statistical Package for the Social Sciences (SPSS) version 25. Data was 
summarized using mean and standard deviation (SD) in quantitative data and using frequency (count) and relative frequency (percentage) for categorical data. Comparisons between non parametric quantitative variables were done using Wilcoxon rank sum test and unpaired $t$ test, whenever the results are similar; the results of the unpaired $t$ test are reported as it is more powerful test. For comparing categorical data, Chi square $\left(\chi^{2}\right)$ test was performed. Exact test was used instead when the expected frequency is less than 5. P-values less than 0.05 were considered as statistically significant.

\section{Surgical technique:}

\section{Group A:}

All procedures were performed under general anesthesia. Perforation's edges were refreshed by Rosen needle. Local infiltration of the post-auricular area and posterior canal skin was done using 2\% Lidocaine with 1:100,000 Adrenaline. Postauricular incision was performed; dissection of post-auricular tissues was continued till the periosteum overlying the mastoid cortex. Scalpel (number 15) blade was used for harvesting a rectangle of periosteum covering the mastoid cortex (Figure 1) just posterior to the ear canal (about 10x15 mm or according to the size of the perforation). Dissection of the posterior canal skin was continued till exposure of the middle ear cavity. Graft was positioned in underlay fashion and the posterior canal skin flap was returned back. Gelfoam squares were used to stabilize the graft. Finally the skin incision was closed and a piece of Vaseline gauze was inserted through the external auditory canal.

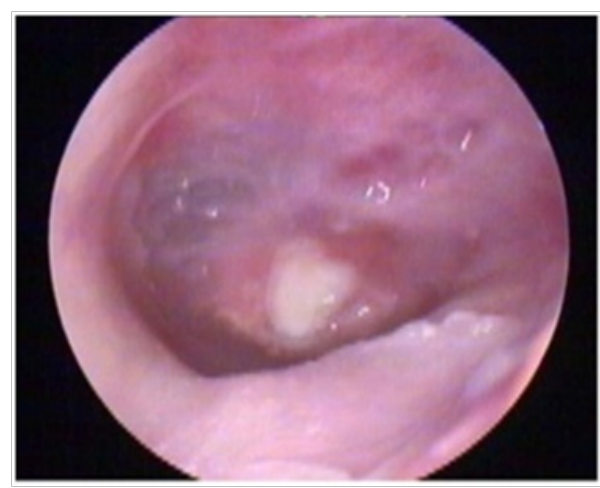

Figure I Periosteal graft harvesting

\section{Group B}

Under general anesthesia, trimming of perforation's edges and infiltration of tragal skin with Lidocaine and Adrenaline were done as above. A $1.5-\mathrm{cm}$ skin incision was made $2 \mathrm{~mm}$ posterior to the tragal cartilage free edge using a scalpel blade (number 11). The subcutaneous tissue was dissected from the perichondrium. Graft was harvested leaving 1-2 $\mathrm{mm}$ of the outer rim of the cartilage for cosmetic purpose. Graft size was approximately one and half the size of the perforation after trimming (Figure 2). Bleeding was controlled. The incision was sutured.
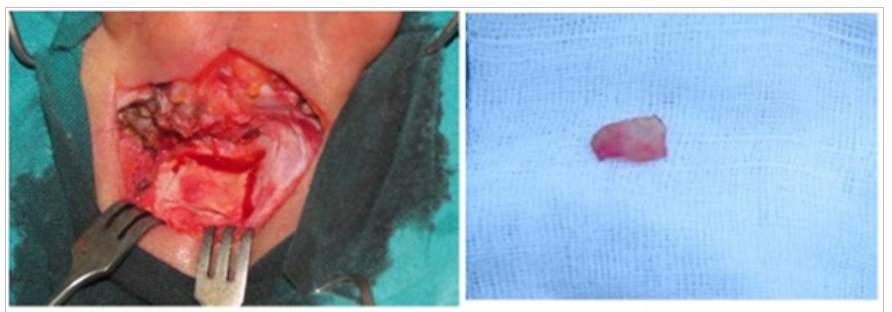

Figure 2 Illustration of the cartilage graft preparation
Using a number 15 blade, a layer of the cartilage with the overlying perichondrium was elevated from all the edges of the graft, kept attached to the remaining part of the graft at its center and kept to dry in this position "Lotus-shaped" or "Butterfly-shaped".

The graft was held with a forceps where the cartilage proper positioned on the undersurface, passed through the perforation to cover the medial surface of the drum while the upper layer (cartilage and perichondrium), covered the lateral surface of the tympanic membrane (sandwich-like) (Figure 3).

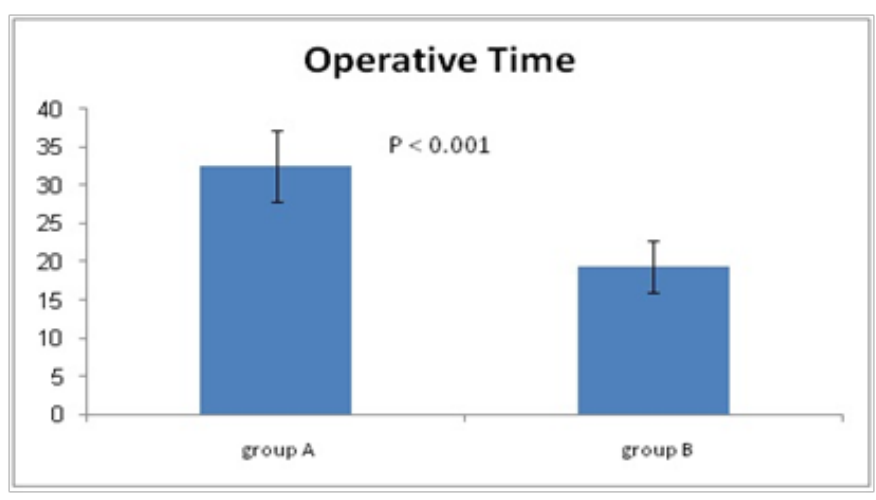

Figure 3 Cartilage graft being placed inlay with the perichondrium on the outer surface

The technique was totally permeatal. Gel foam was placed and the ear was covered by dressings.

Postoperatively, patients had overnight stay, Amoxicillin Clavulonic acid $1 \mathrm{gm}$ twice daily and Paracetamol $500 \mathrm{mg}$ every 8 hours for 1 week were prescribed. The patients were advised to avoid straining or water entry inside the ear. Dressing and stitches were removed 1 week postoperatively. Examination of the ear was performed in the outpatient clinic every week for 1 month and every month for 6 months using otoscopy or otoendoscopy for evaluation of graft take and to detect any external canal or the middle ear infection.

\section{Results}

Considering group A to which underlay periosteal graft was used, 82 cases (out of 88 ) showed complete graft healing representing $93 \%$, while in group B cases where inlay cartilage graft was used, 56 cases (out of 61) had complete graft healing representing 92\% with statistically insignificant P value of 0.8 (Figure 4), (Table 1).

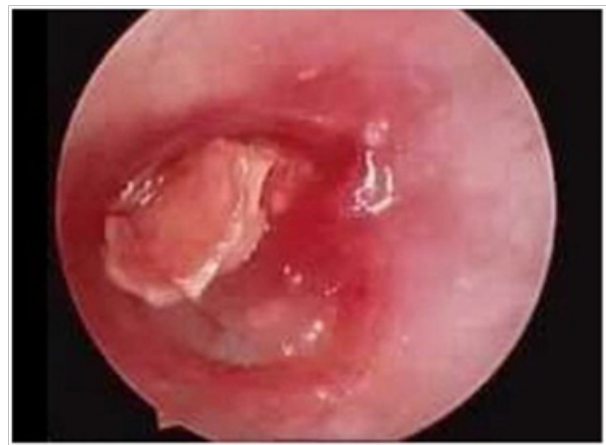

Figure 4 Six months postoperative photo of cartilage graft showing good graft take

In group $\mathrm{A}$, preoperative mean $\mathrm{ABG}$ (Air Bone Gap) of the group was $18 \mathrm{~dB} \pm 5 \mathrm{SD}$ while 6 months postoperative mean ABG was 7 $\mathrm{dB} \pm 5 \mathrm{SD}$ with a mean difference of $-11 \mathrm{~dB} \pm 5 \mathrm{SD}$. If we now turn 
to group $\mathrm{B}$, preoperative mean $\mathrm{ABG}$ of the group was $21 \mathrm{~dB} \pm 6 \mathrm{SD}$ while 6 months postoperative mean $\mathrm{ABG}$ was $12 \mathrm{~dB} \pm 9 \mathrm{SD}$ with a mean difference of $-9 \mathrm{~dB} \pm 7 \mathrm{SD}$ (Figure 5). Mean difference between both groups showed statistical significance with $\mathrm{P}$ value of 0.01 (Table 2).

Table I Comparison between both groups regarding the graft take and operative time.

\begin{tabular}{llll}
\hline & Group A & Group B & P value \\
\hline Graft takes (\%) & 93 & 92 & 0.8 \\
Operative time (min) & $33 \pm 5$ SD & $19 \pm 3$ SD & $<0.001$ \\
\hline
\end{tabular}

SD: Standard Deviation

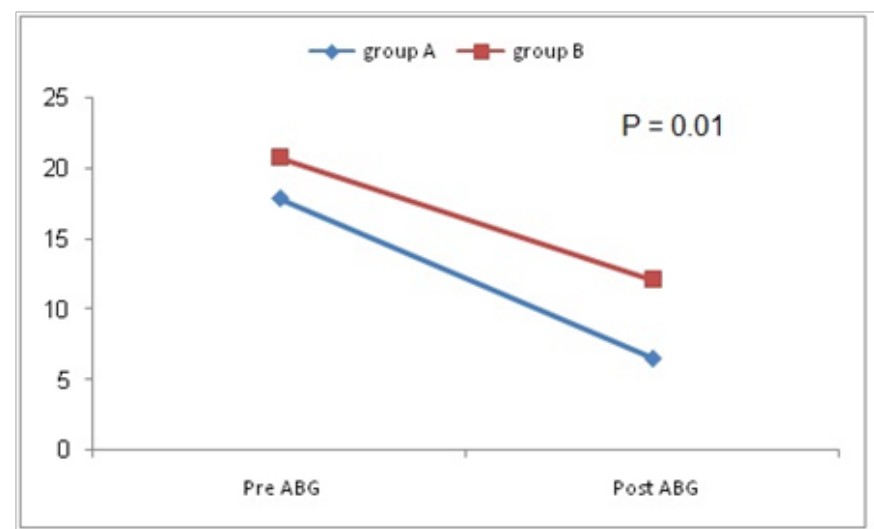

Figure 5 Preoperative and postoperative mean Air Bone Gap changes in both groups

Table 2 Comparison between both groups regarding the mean Air Bone Gap (ABG) difference. SD: Standard Deviation.

\begin{tabular}{llllll}
\hline & Group A & \multicolumn{3}{l}{ Group B } \\
\hline & Mean & SD & Mean & SD & P value \\
\hline ABG Differnce (dB) & $-1 \mathrm{I}$ & 5 & -9 & 7 & 0.01 \\
\hline
\end{tabular}

Mean operative duration in group A was 33 minutes \pm 5 SD while in group B was 19 minutes \pm 3 SD showing statistical significance with $\mathrm{P}$ value $<0.001$ (Table1), (Figure 6).

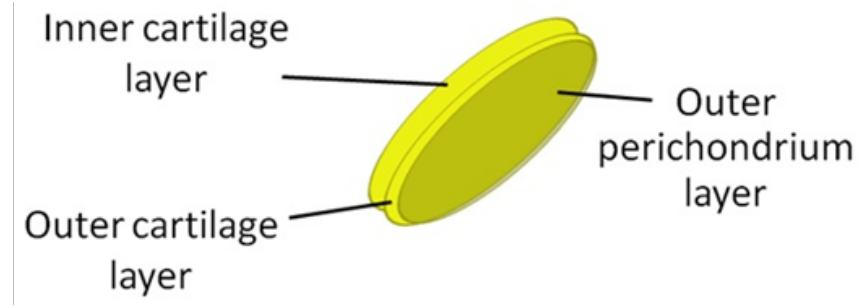

Figure 6 Operative time in both groups

\section{Discussion}

Cartilage grafts have been noted to be well accepted by the middle ear and can survive for long time. ${ }^{7,8}$ Cartilage perichondrial autografts receive nutrition by diffusion and well incorporated in the ear drum. ${ }^{9}$

Chiossone ${ }^{10}$, in his study "Periosteal Grafting in Tympanoplasty", described several advantages of periosteal grafts; being similar to the middle fibrous layer of the ear drum allows it to stick stongly to the malleus handle and the external auditory canal, periosteum can withstand low nutritional status thus it can tolerate the first period after transplantation, and periosteal grafts pliability allow easy handling; also the ample amount and easy harvesting add more to its advantages. ${ }^{11}$

These results matched our study results where both types of grafts showed high success rate of $93 \%$ (group A) and $92 \%$ (group B) with no statistical significant $\mathrm{P}$ value.

Increased ear drum mass and rigiditiy might adversely affect the hearing outcome..$^{12,13}$

Similarly, in this study group A cases (which was subjected to underlay periosteal graft) had better hearing results with mean difference of $-11 \mathrm{~dB} \pm 5 \mathrm{SD}$ compared to group $\mathrm{B}$ cases, where inlay cartilage grafting was used which had a mean difference of -9 $\mathrm{dB} \pm 7 \mathrm{SD}$ with statistically significant $\mathrm{P}$ value of 0.01 . However, Yang et al., ${ }^{14}$ in 2016, concluded in a meta-analysis retrospective study that 1 year follow up of patients underwent full thickness type I cartilage tympanoplasty had better hearing than others underwent tympanoplasty using temporalis fascia. Yang's study conclusion gave the rise to question about the long term hearing results of cartilage tympanoplasty which seems to be better on the long run.

Inlay transcanal cartilage myringoplasty has many advantages: no post auricular incision, no tympanomeatal flap, no ear packing post operatively as the graft gains support from its own stiffness, minimal pain, one day surgery where patient can leave hospital the next day after the surgery or even right after recovery from anesthesia, shorter operative time (mean $=19$ minutes $\pm 3 \mathrm{SD}$ ), and lower costs. Lastly the patient can return back to work, school on the next day after surgery. We recommend long term follow up for future studies as shortage of long term data was a limitation in this study.

\section{Conclusion}

Underlay periosteal and inlay cartilage grafts show high rates of graft take, with better hearing results using underlay periosteal grafts but shorter operative time with inlay cartilage grafts.

\section{Compliance with ethical standards}

Ethical approval: All procedures performed in this study were in accordance with the ethical standards of the institiutional and / or national research committee and with the 1964 Helsinki declaration and its later amendments or comparable eithical standards.

Informed consent: Informed consent was obtained from all individual participants included in the study.

Funding: No fund was received.

Conflicts of interest: The authors have no conflicts of interest.

\section{Acknowledgements}

None.

\section{Conflict of interest}

The authors declare that they have no conflict of interest.

\section{References}

1. Smyth G. Tympanic reconstruction. Fifteen year report on tympanoplasty. Part II. J Laryngol Otol. 1976;90(8):713-741.

2. Wullstein H. Functional middle ear surgery with the aid of free graft. Arch Ohr Nas Kehlkopfheilk. 1952;161:422-435. 
3. Zollner F. The principles of plastic surgery of the sound conducting apparatus. J Laryngol Otol. 1955;69(10):637-652.

4. Eavey R. Inlay tympanoplasty: Cartilage butterfly technique. Laryngoscope. 1998;108:657-661.

5. Rizer FM. Overlay Versus Underlay Tympanoplasty Part I: Historical Review of the Literature. Laryngoscope. 1997;107(12):1-25.

6. Mancini F, Russo A, Sanna M. Grafting techniques for tympanoplasty. Operative techniques in Otolaryngology, Head and Neck surgery. 1996;7(1):34-37.

7. Don A, Linthicum F. The fate of cartilage grafts for ossicular reconstruction in tympanoplasty. Ann Otol Rhinol Laryngol.1975;84:187-191.

8. Glasscock ME, Jackson CG, Nissen AJ, et al. Postauricular undersurface tympanic membrane grafting: a follow-up report. Laryngoscope. 1982;92(7 Pt 1):718-727.
9. Yung M. Cartilage Tympanoplasty. Literature Review. J Laryngol Otol. 2008;122(7):663-672.

10. Chiossone E. Periosteal Grafing in Tympanoplasty. A Preliminary Report. Arch Otolaryngol. 1964;9:302-311.

11. Schlosser W, Pratt L. An Evaluation of Various Tympanoplasty Techniques. Arch Otolaryngol. 1961;74(4):429-434.

12. Dornhoffer J. Hearing results with cartilage tympanoplasty. Laryngoscope. 1997;107(8):1094-1099.

13. Murbe D, Zahnert T, Bornitz M, et al. Acoustic properties of different cartilage reconstruction techniques of the tympanicmembrane. Laryngoscope. 2002;112(10):1769-1776.

14. Yang T, Wu X, Peng X, et al. Comparison of cartilage graft and fascia in type 1 tympanoplasty: systematic review and meta-analysis. Acta Oto-Laryngologica. 2016;136(11):1085-1090. 